\title{
Communications for AnyPLACE: A Smart Metering Platform with Management and Control Functionalities
}

\author{
Dominik Henneke ${ }^{1}$, Christian Freudenmann ${ }^{5}$, Markus Kammerstetter ${ }^{2}$, David \\ $\mathrm{Rua}^{4}$, Lukasz Wisniewski ${ }^{1}$ and Jurgen Jasperneite" 1,3 \\ ${ }^{1}$ inIT - Institute Industrial IT, OWL University of Applied Sciences, 32657 Lemgo, Germany \\ dominik.henneke@hs-owl.de, lukasz.wisniewski@hs-owl.de,juergen.jasperneite@hs-owl.de \\ ${ }^{2}$ Secure Systems Lab, Automation Systems Group, Vienna University of Technology, 1040 Vienna, Austria \\ ${ }^{3}$ Fraunhofer IOSB-INA Application Center Industrial Automation, 32657 Lemgo, Germany \\ ${ }^{4}$ INESC TEC - INESC Technology and Science, 4200 Porto, Portugal ${ }^{5}$ Power \\ Plus Communications AG, 68167 Mannheim, Germany
}

\begin{abstract}
Recent developments under the term Smart Grid change how users consume electricity and interact with the power grid. Smart metering and energy management are developments that transform the yet passive energy consumer to a participant that is actively involved in the energy market by using variable energy tariffs or by demand-response services. But such functionality demands a platform that integrates all smart devices in the users property, connects to external services and electricity providers, and has interfaces that provide information and control to the user. AnyPLACE will develop such platform. Based on the latest legislation in the European member states, it will incorporate smart meters and create links to external service providers. Furthermore, it connects the devices in the property of the end-user in order to be able to fully monitor and control the energy consumption. This paper presents the AnyPLACE idea and the problems that are solved on the communications aspect. It provides an in-depth analysis of current European legislation in the context of smart metering and provides the requirements that need to be realized by the platform. Additionally, it proposes a strategy to create a solution that can be used in any place of Europe. The paper also incorporates the security and privacy requirements in different domains and sketches a solution and architecture to fulfill these by incorporating existing open source implementations as provided by the openHABB project.
\end{abstract}

\section{INTRODUCTION}

The advent of the Smart Grid concept has been changing the paradigm of the electric industry, the way different stakeholders interact with the electric grid, and how different energy services can be exchanged among them. Included in those stakeholders are the consumers, which are no longer mere passive agents in the system. Instead, they are likely becoming active participants with an important role in using their flexibility in the provision of new services (ex: demand-response) which can be exchanged with other stakeholders (ex: retailers or system operators); they are even expected to be capable of optimizing their energy use on their own.

The challenge in having these end-users involved in energy management is multidimensional and affects areas such as awareness, education, and empowerment. The technology has a key role in supporting these areas by providing an energy management platform that can be used in a simple manner and allows end-users to derive benefits from the optimization of energy use. It needs to collect information in a mostly automated fashion, provide easy access to information, and be capable of interacting with devices and systems.

AnyPLACE is a $\mathrm{H} 2020$ project that intends to implement a modular, adaptable, and cost-effective solution to support the energy management of households and similar buildings, and integrates smart metering platforms. By using the flexibility of loads and potential microgeneration systems, the objective of the project is to demonstrate the feasibility of implementing advanced monitoring and control schemes that - by including comfort preferences - allow the end-user to better manage the use of energy. The project intends to design, implement, and test hardware and software modules that when combined will produce different solutions that are capable of being continuously used by end-users in what it is expected to become a "natural" way of managing the energy use. A detailed characterization of the physical, regulatory, and market contexts from five different EU countries is required to evaluate the applicability of an energy management solution and to interact with smart metering. This information was used to establish the definition of the AnyPLACE platform and the interactions between end-users, devices, and systems.

The aim of the project is not to define new communications standards or regulation proposals. The main contribution is the definition of a flexible and extendable architecture that combines existing communication protocols and smart metering regulations into a solution that can be used in any place of Europe. The contributions of this work are a recent study of smart metering regulations in different European countries as well as the definition of an approach to cover all these in a single modular approach. A service-oriented approach will be presented that provides the basis for a state-of-the-art energy management and smart metering platform. Furthermore, 
it presents what security aspects need to be taken into account and how these can be implemented in the proposed solution.

Section II presents existing solutions and previous projects. Section III further details the AnyPLACE approach and supporting methodologies and section IV focuses on the requirements that are put from the communications perspective - both for communications with external stakeholders as well as with appliances. Section V presents the architecture that AnyPLACE proposes to fulfill the requirements and to implement a suitable solution. Finally, section VI concludes the paper and provides an outlook about the future activities in the project.

\section{RELATED WORK}

Several projects already covered the areas of energy management, smart meter integration, and end-user engagement. The OPENmeter project addressed the specification of a set of open standards to be used on an Advanced Metering

Infrastructure (AMI) supporting electricity, gas, waster, and heat metering [1]. It is focused on the distribution network and not on the end user interactions. Also the followup project Meter-ON doesn't address this [2]. The OpenNode project proposed an architecture that combines smart metering with grid automation to enable a reliable and efficient grid operation [3]. The specified solution followed a similar target, but without the strong focus on integrating the different country-specific regulations on smart grid integration [4]. The ADDRESS project studied, developed, and validated solutions to enable an active demand and exploit its benefits [5]. It also defined communications requirements but was not focused on integrating the different domains of internal and external communicaions [6]. The Smart City Mannheim project integrated an energy mangement tool to households and connected the households via powerline to the smart grid. This enabled customers to react on variable prices on basis of a market place and thus participate in the stabilization of the distribution grid [7]. Energy@Home targeted the increase of energy efficiency of a house through the information exchange related to energy usage, energy consumption, and energy tariffs between smart devices and domestic appliances [8]. Smart House/Smart Grid validated an Information and Communication Technology (ICT)-enabled collaborative technicalcommercial aggregation of smart houses to provide higher levels of energy efficiency [9].

The problem of integrating different energy consuming devices to a single platform in order to enable a sophisticated energy management has been reviewed and also solved by a number works. There are already a large number of energy management systems for smart homes in the market. Qivicon, NEST, Miele@home, or Bosch SmartHome are examples for commercial solutions. OpenHAB or Eclipse SmartHome are open source alternatives. However, all these solutions are often limited to support devices of a single vendor, are not able to integrate recent smart metering technologies, or are also often expensive.
In the literature, Koß et al. propose the use of a layered Service-oriented Architecture (SoA)-based distributed architecture with open interfaces and support for plug-and-play hardware and software components [10]. They deployed and tested their development in a 'Smart Living Lab'. Irlbeck et al. presented a service-oriented reference architecture for different use-cases in the smart grid scenario [11]. Shrestha et al. proposed a method to integrate different domain-specific applications into a Internet of Things (IoT) [12]. They used the IoT standard Quantum Lifecycle Management (QLM) to abstract different domains such as smart energy, smart building, or smart industry in order to enable a communication between them. They used openHAB as example for the smart building domain and extended it with the proposed interfaces. Granzer and Kastner proposed a mapping between different technologies in the scope of building automation systems and a technological-independent data representation [13]. As example, they mapped KNX, ZigBee, and other protocols to an OPC UA representation.

The topic of energy management and smart grid communications for the end user is a recent topic that has been analyzed and implemented by a number of commercial solutions and research projects, but the presented projects either provide outdated information on e.g. smart metering-due to the latest regulations in the EU member states-or do not provide a comparable feature set. Furthermore, they are not as flexible and extensible as required or are not directly reusable for the implementation of the project due to missing implementations or support.

\section{THE ANYPLACE APPROACH}

The implementation of a solution such as AnyPLACE depends on a design that is suitable for controlling loads and microgeneration, integrating smart meters, and engaging endusers in exploring potential advantages in optimizing their energy use. The paradox is that the technology already allows this ambitious implementation, since a significant number of existing solutions already accomplishes parts of these features. However, a cost-effective solution that integrates all these domains, is easy to use, and complies with smart metering regulations is clearly missing.

The AnyPLACE approach has initially been contextualized within the Smart Grid Architecture Model (SGAM) methodology [14], where a business use case was established with interactions among different stakeholders. Different use cases such as Ancillary Services, Retail Market, and Customer Energy Management are provided to end-users, Distribution System Operators (DSOs), Transmission System Operators (TSOs), or manufacturers. Based on these findings, a set of requirements was established as part of the functional specification of the platform. Sets of functionalities were established under the groups of Energy Management, End-user Interaction, ICT, and Maintenance and Support.

The set of services to be supported by the AnyPLACE platform followed from the definition of high-level use cases in these groups. The energy management group defines the services Data Analytics and Energy Awareness, Tariff 
Selection Management, Local Energy Management, and Demand Response. Services for the end-user interaction group are Preferences and Configurations, as well as Media Presentation Management. The ICT group defines the services Local Device Management, Interaction with Stakeholders, Metering Management, Systems Integration and Management, Cloud Services, and Secure Access and Exchange. Finally, the maintenance and support group defines the services Maintenance Services, Reporting and Alarms, and Storage Management.

Based on these four types of services, functional requirements were identified which, along with the expected inputs and outputs, helped in bounding the AnyPLACE specification. This creates a component layer according to SGAM guidelines. Concerning ICT-also referred to as communications - a segmentation was introduced in order to cover the multiple interactions that are required. Requirements were defined separately for the vertical interactions with local devices and systems (ex: appliances, sensors, media devices, Heating, Ventilating and Air-Conditioning (HVAC)), meters (ex: legacy, smart), remote systems (ex: web-services, cloud), and transversely through the secure access and data exchange (ex: authentication, authorization, access control, confidentiality, protection). These interactions can be combined into external communications (with meters and remote systems) and internal communications (with local devices and systems).

In consequence of this analysis, Fig. 1 shows the proposed modular reference architecture and depicts the different modules that are to be included in the AnyPLACE solution: The AnyPLACE Central Module is the core of the architecture and provides the main functionality of the identified requirements. It is extended with basic and advanced modules, that provide the interaction with different devices and stakeholders, as well as with the end-user. The basic modules are expected to be available in all AnyPLACE installations while the advanced modules can be incorporated depending on the availability of devices in the end-users premises, services provided by independent stakeholders, or available budget for the platform. This approach permits the development of a modular and adaptable infrastructure that is likely to be suited for a large group of different end-users.

From the viewpoint of security and data privacy, AnyPLACE puts high requirements on the architecture of the platform. These requirements start on the definition of different user roles that can use the platform. Regular users are only allowed to view data while advanced users can configure the platform or switch devices. Another requirement concerns the permissions of the internal modules and algorithms. Based on the principle of least privileges, each part should only have the smallest set of abilities that are needed to perform their respective tasks. For example an algorithm for weather forecast is not authorized to change the operation state of a smart plug or a heating optimization is not authorized to switch the room lights or access detailed energy statistics.

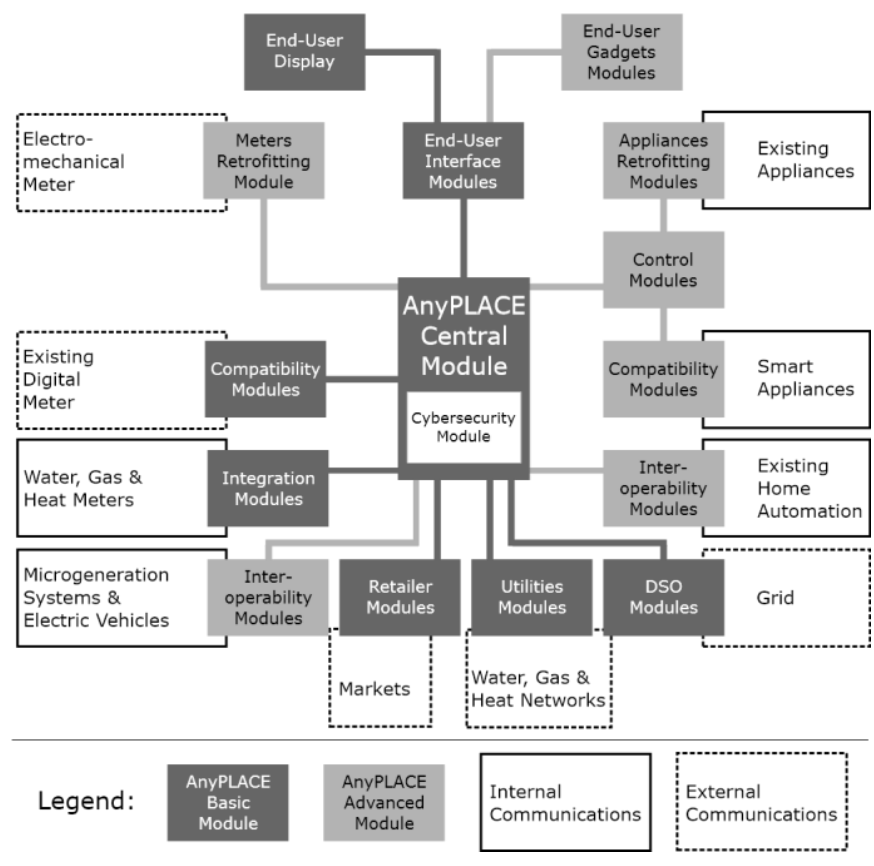

Fig. 1. Initial design of the modular architecture for AnyPLACE.

\section{COMMUNICATIONS REQUIREMENTS}

AnyPLACE communications consists of internal as well as external communications as depicted in Fig. 1. Internal communications is defined as the communications with devices in property of the end-user (ex: smart devices, electric vehicle charging stations, private submeters). External communications on the other hand concerns the communications with actors, services, and components that are not part of the end-user property (ex: distribution system operator, meters of the meter system operator). The AnyPLACE project identifies, analyzes, and develops solutions for both sectors. As a preparation for this purpose, communications requirements were defined at a technical and technological level.

The major sources to derive those requirements are the planned functionality, the physical context, and the regulatory context. Of those sources, the physical and regulatory context may differ from one country to another. However, due to the European context of the project, the developed solution should be able to be situated in any place within Europe. For this purpose, in order to avoid the development of different products from the very beginning, an approach is necessary to identify a common basis that can be situated in any country and may only be adapted depending on country specific requirements.

In general, there are three approaches to achieve this: The first develops a solution that suits one country that has highly detailed mandatory communications and security requirements. Conflicting requirements of other countries are handled in modifications to derive a solution. The second approach develops a solution for every country separately and derives a common basis afterwards. The third approach combines the advantages of the other approaches and analyzes the mandatory requirements of all countries and derives a common basis from 
that which is then being developed as a common solution. This approach — which has been chosen for the project-is depicted in Fig. 2.

\section{A. External Communications}

Concerning external communications, the regulatory context has very different extents in different European countries. There are several documents issued by the EU that demand general requirements to provide final customers with times pecific information about their energy consumption or to ensure data privacy and data security (e.g. [15]). However, they leave plenty of room for the member countries to further specify the regulations of the technology used for the data transfer. The following section provides details of the country specific mandatory communications requirements in Belgium, Portugal, Netherlands, Germany, and Austria.

Belgium has not specified requirements on this topic yet. In Portugal, the EU directive is introduced via Portaria 231/2013 [16]. There are also highly detailed industry-driven standards on smart meters. As far as the requirements of an interaction between smart meters and a platform such as AnyPLACE is concerned, all Portuguese smart meters mandatorily provide an interface to be interconnected with a Home Area Network and a display. The Netherlands Technical Agreement (NTA 8130) [17], [18], as a main part of the Dutch regulations, describes the communications architecture of the Dutch smart meter environment in which the electricity meter has a communications module that serves as a gateway for other meters. This gateway needs to provide an interface where final customers can read meter data from. Additionally, it has an external interface which can only establish a connection to a central system. Authorized external market entities such as the grid operator, suppliers, or independent service providers can use this connection as proxy. This approach is similar to the German regulations that demands a Smart Meter Gateway (SMGW) that is placed on an electricity meter and can be connected to several meters and-among other interfacesprovides an interface to the final customer to read out meter data [19], [20]. Likewise to the Dutch central server, there is the Gateway Administrator (GWA), responsible to provide secure communications between the external interface of the gateway and external market entities (e.g. to provide meter data or to manage controllable devices in the final customer's premises).
The communications between the gateway, meters, controllable local systems, and external market entities is specified in technological detail in respective technical guidelines [21]. A special remark can be made on the data log which is demanded to enable the final customer to be aware of the data exchanged via the gateway. Austria as a final example, also demands to provide the final customer with detailed information about the usage of his or her data. Likewise to the German approach, secured and encrypted communication is demanded, although — in the Austrian set of regulations - without giving technological specifications.

The AnyPLACE solution includes and controls many critical assets such as metering data or controllable loads. Depending on the type of asset, different security measures are not only necessary but may also be mandated by EU-member states. For instance, for handling smart metering data, both the Netherlands [22] as well as Germany [19], [23] have extensive security requirements for the employed hardware, software, and communications protocols. These include strong cryptographic authentication, end-to-end security, the use of a Public Key Infrastructure (PKI), and key storage on tamper resistant smart cards. While metering data has a strong privacy focus, controllable loads are security critical as they can affect the stability of the power grid at a large scale. If an attacker manages to switch a large number of high current controllable loads (a boiler or building heating) at the same time, the sudden load change can cause critical voltage peaks outside the normal grid operating range. Potentially even amplified by the utilities' protection switches, the result of the attack could be a chain reaction leading to a blackout and physical damages in the worst case. The security design for AnyPLACE is based on a thorough analysis of the hardware, software, and communications security requirements mandated by the EU-member states for smart metering and critical infrastructures in general. Over external interfaces the solution always acts as a network client to reduce the attack surface. Any communications with external services adheres to the extensive smart metering security requirements including certificate based strong mutual authentication, end-to-end security, PKIusage, and key storage, key and random number generation, or signature generation and verification on a tamper resistant smart card.

\section{B. Internal Communications}

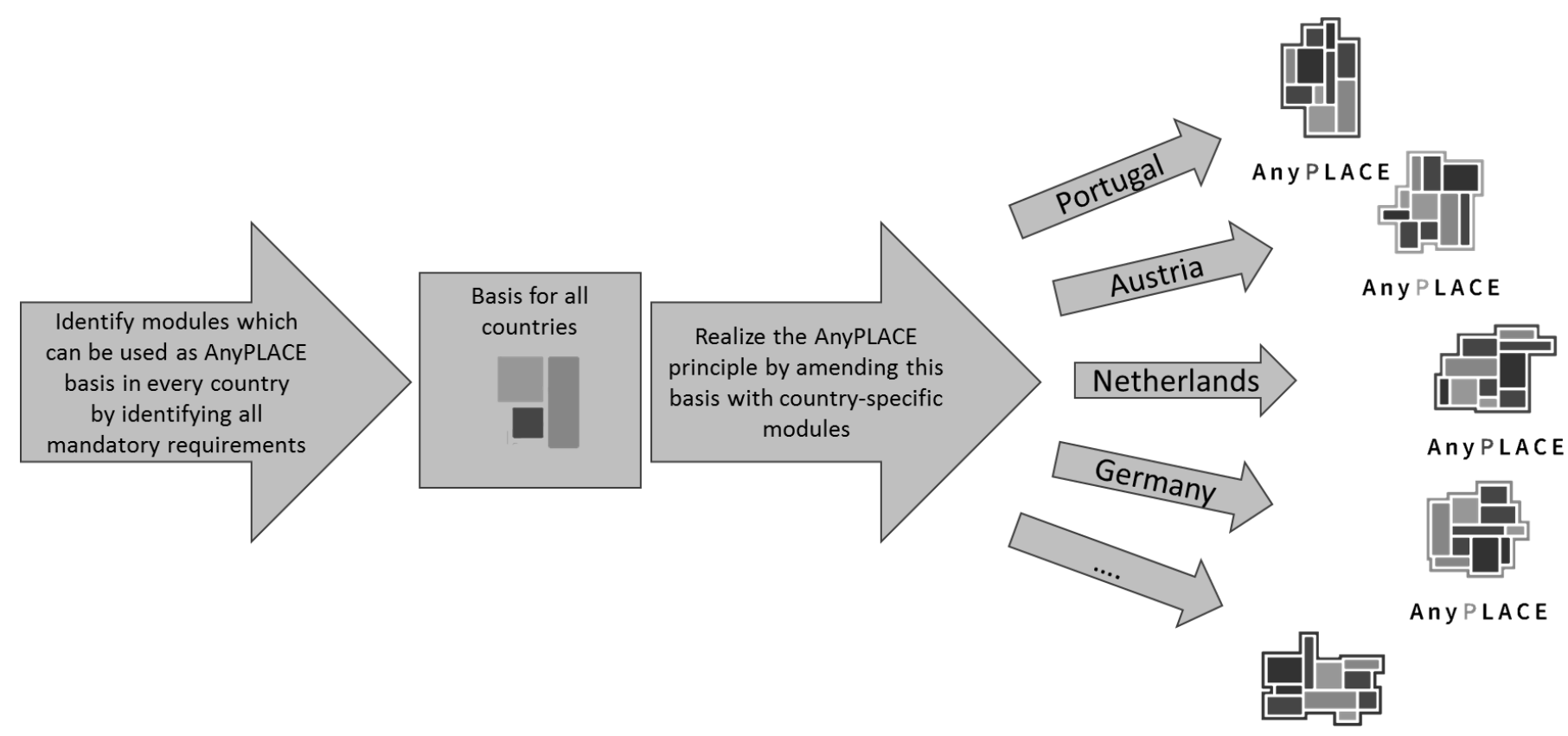


Internal communications covers the connection to devices in the customers premises. These include controllable or uncontrollable devices for power generation or consumption. Under the term 'smart home', an increasing number of devices that enter the market today are equipped with communications interfaces. But due to the diversity of existing (wired and wireless) connection technologies and a large number of competing standards, the devices are not compatible to each other. In fact, the current implementations of many vendors force users into vendor-dependent ecosystems. In order to be able to implement energy management algorithms that can visualize and control the device states, there is a need to access and control all these different technologies and protocols (including popular wired and wireless protocols such as KNX, $\mathrm{Wi}-\mathrm{Fi}$, ZigBee, or Bluetooth, as well as a huge variety of different protocols). The challenge in creating the universal communications modules for all different technologies is to have on the one side the necessary interfaces on the hardware platform and on the other side to provide the implementations to all potential protocols of the devices that need to be supported. However, many communications details are neither publicly available nor intended to be used by a third-party. Thus, instead of a custom implementation of the protocols, the reuse of already existing implementations is preferred, if conforming with the requirements and regulations.

For internal communications in AnyPLACE, the solution leverages the concepts of the strong security architecture for external communications interfaces whenever possible. Due to the vendor-specific and proprietary protocols for smart home devices, the full extent of the AnyPLACE security architecture is not applicable. Communications security can then only be realized in a best effort manner by relying on the security measures in the vendor's protocols and the utilized communication technologies. Nevertheless, the hardware and software security mechanisms as well as a user centric authorization scheme within the AnyPLACE solution can still effectively improve the overall security of these smart home devices. In addition, it is important to consider the physical communication technologies. While utilizing an insecure protocol over a wired interface to communicate with a smart home device within the user's premises just a few meters away will typically not have a considerable security impact, using the very same protocol over an unprotected wireless link is a different scenario. In this case an attacker could easily connect or otherwise interfere with the wireless link, the smart home service, and the linked AnyPLACE services. For wireless interfaces such as Wi-Fi, ZigBee, or Bluetooth the use of integrated link-level security modes is thus highly important as well.

\section{ARChiteCtUAL DESIGN}

The architectural design follows from the combination of the functional requirements, the regulatory mandates, and the technological properties and limitations. The following sections present different aspects for the implementation of AnyPLACE. An SoA-approach and a security concept are presented and the exemplary analysis of an existing energy management framework is carried out. A main focus on the project lies on reusing existing implementations and frameworks if possible. Thus, in the first step a strategy is developed on how to integrate an existing framework in the solution. In the next step, a possible framework is evaluated and the overall application architecture is specified.

\section{A. Service-oriented Architecture}

To face the communications requirements of the external and the internal domains, the use of an SoA is aimed. Different connections and interfaces to the domain of external devices and entities are abstracted to weakly coupled services which are consumed by the energy management and user interaction parts of the project. A core aim of AnyPLACE is to achieve a working prototype in a reasonable amount of time and at an affordable price. Section II shows that already a large number of works, projects, and implementations exist that faced these problems before. Therefore, it is highly recommended to think about integrating these existing approaches into the platform.

Thus, assuming existing implementations can be reused, three different strategies were identified to realize the communications SoA in AnyPLACE (Fig. 3): greenfield, middleware, and framework. The greenfield approach implements the energy management platform, as well as the SoA and all required interfaces to support communications with external and internal entities. It releases a fully customized solution that fits to all requirements on the downside of being highly time consuming and lacking reusability. The middleware approach implements the energy management platform, but reuses the

\begin{tabular}{|c|c|c|c|}
\hline Application & & AnyPLACE & AnyPLACE \\
\hline \hline Middleware & AnyPLACE & $\begin{array}{l}\text { Existing } \\
\text { Framework }\end{array}$ \\
\hline & $\begin{array}{l}\text { Existing } \\
\text { Framework }\end{array}$ & \\
\hline Devices / Services & Devices / Services & Devices / Services \\
\hline a) greenfield & b) middleware & c) framework
\end{tabular}

Fig. 3. Three alternatives to realize the SoA: The greenfield approach a) to implement the complete architecture. The middleware approach b) to reuse the implementations of an existing framework. The framework approach c) to extend an existing framework with the needed functionality.

SoA of an existing (open source) framework. This framework already implements most of the communications requirements and can be extended with missing features. Therefore, the development effort is reduced and-depending on the realized architecture - the framework can be independently updated, changed, or replaced if necessary. The access from the AnyPLACE platform to the SoA of the framework can either be realized by using the Application Programming Interfaces (APIs) that are provided by the framework or are specified and implemented according to the needs of the project. The framework approach fully reuses an existing (open source) framework that provides the implementation platform for the 
energy management as well as the SoA. The framework is extended with all the functionality that is required to realize the intended solution. The main effort will be to ensure that all required functions can be implemented in the platform. As the framework already provides the communication interfaces for a large number of devices-especially from the in-building domain - only yet unsupported devices and services need to be implemented.

TABLE I

CORE RESULTS OF THE SWOT-ANALYSIS FOR EACH APPROACH

\begin{tabular}{llll}
\hline & Greenfield & Middleware & Framework \\
\hline Strength & Customization & Reuse & Maturity \\
Weakness & Maturity & Integration & Limitation \\
Opportunity & Tailor-made & Decoupling & Effort \\
Threat & Effort & Design & Dependency \\
\hline
\end{tabular}

The results of a Strengths, Weaknesses, Opportunities, and Threats (SWOT)-analysis for each approach are provided in TABLE I. While the greenfield approach provides the most flexibility and an opportunity for a tailor-made design, the maturity is limited and effort of such a solution is high. The framework approach counteracts by providing a mature solution and reduced effort, but the potential limitations and the introduction of a huge dependency are critical. The middleware approach reuses the advantages of the framework approach but adds additional decoupling, however, this adds an additional interface and software layer. Furthermore, despite the reduced dependence on the existing framework, architectural design changes by the maintainers will still affect this approach.

\section{B. Review of an Existing Framework: openHAB}

OpenHAB is a vendor and technology agnostic open source automation software for smart homes [24]. It provides a platform that is on the one side able to connect to various smart devices from diverse manufacturers and on the other side creates automation rules to interconnect these. Its main advantage above other (commercial) smart home solutions is its openness that allows to freely create management solutions without vendor lock-in or restrictions on communication interfaces. The framework provides programmability by extending the OSGI-based platform with custom modules and also provides Domain-specific Languages (DSLs). The compatibility to external devices is integrated by bindings that either directly use the hardware interfaces of the platform or communicate with external gateways that provide access to other technologies. The framework already provides more than 100 bindings, including KNX, EnOcean, or Modbus. Additionally, the software provides a RESTful API that can be used to access the devices and all parameters.

A usage in the framework approach is not possible due to the incompatibility of the current openHAB concept with the AnyPLACE requirements (mainly the principle of least privileges). However, a use in the middleware approach is possible as the necessary security and access control requirements can be implemented in a higher layer inside of the AnyPLACE platform.

\section{AnyPLACE Application Framework}

Fig. 4 depicts the proposed application architecture that bases on the described middleware approach. The security requirements are provided by a central Access Control Module that grants access to data and communication to other modules based on the Access Control Policies defined by the end user. External communication is provided to other modules in a secure and confidential manner. To gain high flexibility, modules are stand-alone processes and can therefore be exchanged or run on a physically different machine.

\section{Security}

A working security architecture for AnyPLACE relies on a user centric authorization in order to let the user define, which services are allowed to interact with each other. Considering an energy retailer's web service with variable pricing information and a controllable load within the user's premises, the user would thus set up controllable load switching as follows: Initially, the user would sign up to the energy retailer's service to exchange authentication information (i.e. certificates) and to obtain the URL of the pricing information service. The exchange itself is secured with the help of the PKI so that both the user as well as the energy retailer can be sure that each party is who it claims to be. Once the service access URL is set up in the AnyPLACE solution, it can connect to the service over the external communications interface. This connection is secured with security measures such as mutual authentication using certificates or end-to-end security and, since the AnyPLACE solution acts solely as client, the external

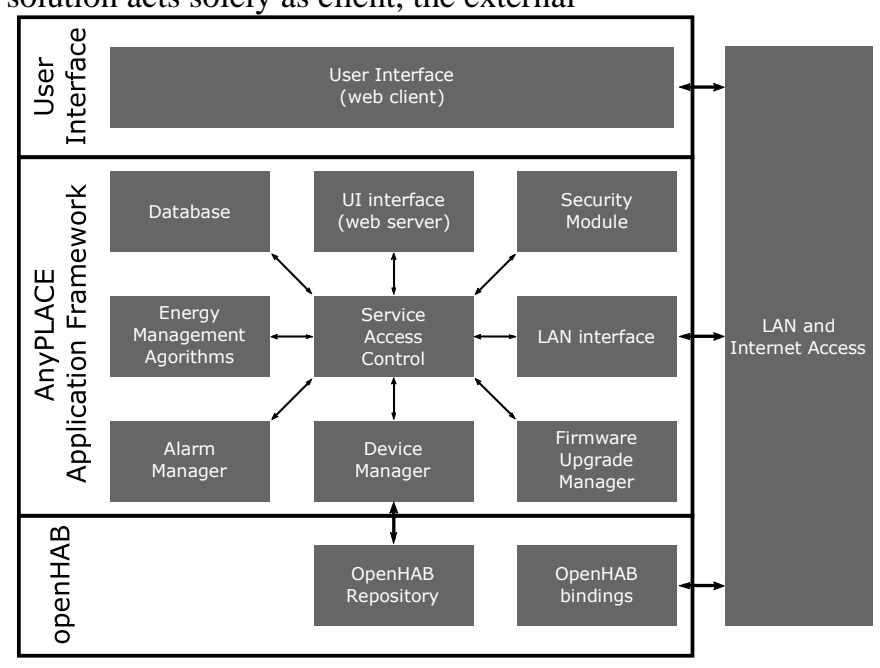

Fig. 4. AnyPLACE Application Architecture.

attack surface is minimized. During setup, the user connects the pricing information service with the controllable load service. During connection setup, the user needs to explicitly authorize the information exchange between those services for the purpose of switching the load. After setup, the solution can thus automatically and securely receive energy pricing information over the external communications interface and react 
accordingly by controlling the controllable load withing the user's premises.

\section{CONCLUSION AND OUTLOOK}

Smart metering and energy management are important topics in future energy systems. The AnyPLACE solution provides a platform that integrates the domains of home energy management and smart metering. This paper provides information about the current legislation in the European member states concerning smart metering-or external communications in general. There are different approaches in the communications architecture and security requirements. While Netherlands and Germany require the use of a secured intermediate gateway that collects data from connected smart meters and communicates them to external services, other countries demand less secured and standardized approaches. Reasons for these differences are the rather superficial EU specifications that apply to such smart metering platform with control and management functionalities. While main contents such as the provision of an interface to the user and the ensured data privacy and data security are defined, the specification of technology and data transfer is left open. The development of a platform such as AnyPLACE that should be usable in all European countries is thus challenging. However, the presented approach on how to tackle these differences shows that only slight modifications are required if the initial design considered this situation.

A lack of universal standards is also highlighted in the internal communications with devices in the end-users property. There is a large amount of communications technologies and protocols that make it hard to build a solution that can be interconnected with all existing devices. However, there has much work already been carried out to solve this problem. The paper presents an approach on how to incorporate this work in AnyPLACE. In the end, a solution is sketched, that fulfills all of the defined requirements from the communications and security perspective. Also, the openHAB framework has been identified as a suitable candidate to use in the implementation.

In future, the information that were gained by the analysis that was carried out and presented in this paper will be used to implement the proposed platform. A realization and test trial in real households in the rural area of Germany will show the influence on energy consumption and use of end-users while using the platform.

\section{ACKNOWLEDGMENT}

This project has received funding from the European Union's Horizon 2020 research and innovation programme under grant agreement No 646580.

\section{REFERENCES}

[1] European Commission. (2008) OPEN meter - Open Public Extended Network metering. Retrieved April, 15 2016. [Online]. Available: http://cordis.europa.eu/project/rcn/101038 en.html

[2] P.-O. Granstrom, M. Baron, R. Denda, and A. Go" $\mathrm{n}^{\sim}$ '1, "Learning from european smart metering deployments," Metering International, no. 4, pp. 22-24, 2014
[3] J. Aguiar, R. Soriano, J. Gotz, F. Lem“ enager, R. Mosshammer, and’ P. Gouveia, "DER and OpenNode: integration of DG in an open architecture for secondary nodes in the smart grid," in Integration of Renewables into the Distribution Grid, CIRED 2012 Workshop, May 2012, pp. 1-4.

[4] F. Lemenager, R. B. Prieto, R. Mosshammer, C. Joannic, M. A. Monferrer, R. Soriano, and N. E. Portero, "Assessment and outlook of the OpenNode smart grid architecture," in Electricity Distribution (CIRED 2013), 22nd International Conference and Exhibition on, June 2013, pp. $1-4$.

[5] R. Belhomme, R. Cerero Real De Asua, G. Valtorta, A. Paice, F. Bouffard, R. Rooth, and A. Losi, "ADDRESS - active demand for the smart grids of the future," in SmartGrids for Distribution, 2008. IETCIRED. CIRED Seminar, June 2008, pp. 1-4.

[6] I. Berganza, E. Lambert, A. D. Paice, R. Napolitano, and A. Sendin, "Communications requirements for smart grids," in 21st International Conference on Electricity Distribution (CIRED 2011), 2011, pp. 1-4.

[7] MVV Energie. (2013) Modellstadt Mannheim (moma). Retrieved April, 17 2016. [Online]. Available: http://www.modellstadt-mannheim.de/

[8] Energy@home. (2016) Energy@Home. Retrieved April, 152016. [Online]. Available: http://www.energy-home.it/

[9] Smarthouse-Smartorid Consortium (2008) SmartHouse/SmartGrid. Retrieved April, 15 2016. [Online]. Available: http://www. smarthouse-smartgrid.eu/

[10] D. Koß, D. Bytschkow, P. K. Gupta, B. Schatz, F. Sellmayr, and“ S. Bauereiß, "Establishing a smart grid node architecture and demonstrator in an office environment using the soa approach," in 2012 International Workshop on Software Engineering for the Smart Grid (SE4SG), June 2012, pp. 8-14.

[11] M. Irlbeck, D. Bytschkow, G. Hackenberg, and V. Koutsoumpas, "Towards a bottom-up development of reference architectures for smart energy systems," in 2013 2nd International Workshop on Software Engineering Challenges for the Smart Grid (SE4SG), May 2013, pp. 9 16.

[12] N. Shrestha, S. Kubler, and K. Framling, "Standardized framework for" integrating domain-specific applications into the iot," in Future Internet of Things and Cloud (FiCloud), 2014 International Conference on, Aug 2014, pp. 124-131.

[13] W. Granzer and W. Kastner, "Information modeling in heterogeneous building automation systems," in Factory Communication Systems (WFCS), 2012 9th IEEE International Workshop on, May 2012, pp. 291300.

[14] Smart Grid Coordination Group (SGCG), "Smart Grid Reference Architecture," CEN-CENELEC-ETSI, Report, November 2012.

[15] The European Parliament and the Council of the European Union, "Directive 2012/27/eu of the european parliament and of the council," Official Journal of the European Union, no. 315, pp. 1-56, November 2012.

[16] Ministerio da economia e do emprego, "Portaria n.' 231/2013," Ministerio da economia e do emprego, Tech. Rep., 2013.

[17] Netbeheer Nederland WG DSMR, "Dutch smart meter requirements - P1 companion standard," Netbeheer Nederland, Tech. Rep., June 2015, version 5.0.1 Final.

[18] _ , "Dutch smart meter requirements - main document," Netbeheer Nederland, Tech. Rep., Mar 2014, version 4.2.2 Final.

[19] Bundesamt fur" Sicherheit in der Informationstechnik (BSI). (2014) Protection Profile for the Gateway of a Smart

Metering System. Retrieved April, 14 2016. [Online]. Available: https://www.commoncriteriaportal.org/files/ppfiles/pp0073b pdf.pdf

[20] Cabinet of Germany, "Entwurf eines Gesetzes zur Digitalisierung der Energiewende," Bundesregierung, Gesetzentwurf der Bundesregierung, 2015.

[21] Bundesamt fur" Sicherheit in der Informationstechnik (BSI), "Anforderungen an die Interoperabilitat der Kommunikationseinheit" eines intelligenten Messsystems," BSI, Technische Richtlinie BSI TR03109-1, March 2013, retrieved April, 14 2016. [Online]. Available: https://www.commoncriteriaportal.org/files/ppfiles/pp0073b pdf.pdf

[22] Netbeheer Nederland, Privacy \& Security Working Group. (2010) Privacy and security of the advanced metering infrastructure. Retrieved April, 14 2016. [Online]. Available: http://hes-standards.org/doc/SC25_ WG1 N1538.pdf 
[23] Bundesamt fur Sicherheit in der Informationstechnik (BSI). (2013)* Protection Profile for the Security Module of a Smart Metering System (Security Module PP). Retrieved April, 14 2016. [Online]. Available: https://www.commoncriteriaportal.org/files/ppfiles/pp0077b pdf.pdf [24] openHAB UG (haftungsbeschrankt. openhab. Retrieved April, 13 2016." [Online]. Available: http://www.openhab.org/

(C) 2016 IEEE. Personal use of this material is permitted. Permission from IEEE must be obtained for all other uses, in any current or future media, including reprinting/republishing this material for advertising or promotional purposes, creating new collective works, for resale or redistribution to servers or lists, or reuse of any copyrighted component of this work in other works. 